\title{
A Minimal Medium for the Cultivation of Infective Trypanosoma cruzi Epimastigotes
}

\author{
By JOSÉ L. AVILA, * ROBERTO PÉREZ-KEPP AND ANTONIO BRETAÑA \\ Instituto Nacional de Dermatología, Apartado 4043, Caracas 1010A, Venezuela
}

(Received 22 March 1982; revised 9 June 1982)

In a culture medium containing bovine liver catalase, but lacking exogenous free amino acids, only two vitamins (choline and folic acid) were found to be essential for the continuous in vitro multiplication of seven different infective strains of Trypanosoma cruzi. The provision of additional vitamins or sugars had no stimulatory effect under these culture conditions. These, and previous studies, have allowed the design of a trypanosomal minimal medium composed only of bovine liver catalase, choline, folic acid, glucose and inorganic salts. This was able to support the continuous cultivation of $T$. cruzi for more than 12 consecutive passages (i.e. about $160 \mathrm{~d}$ of culture). By several criteria, namely morphological features as seen by electron microscopy, infectivity for vertebrate and invertebrate hosts, glucose utilization and protein biosynthesis, this medium (which is the simplest so far described) proved to be nutritionally adequate for Trypanosoma spp. In addition, the medium appeared to be relatively specific for $T$. $c r u z i$, as it did not support the growth of $T$. rangeli and American isolates of leishmania.

\section{INTRODUCTION}

In a previous communication (Avila et al., 1979a) the continuous cultivation of infective epimastigotes of Trypanosoma cruzi in a totally defined culture medium was reported. This liquid medium contained a single purified protein, 8 vitamins, 4 nucleotides, 13 amino acids, glucose and inorganic salts. Among 12 different commercial proteins tested as nutrients, only BLC, horseradish peroxidase, bovine haemoglobin and lactoperoxidase supported continuous growth, at relatively high yield, of mouse-infective $T$. cruzi, while other haemoproteins were inactive. BLC had the best $T$. cruzi growth-promoting activity of all the proteins tested; indeed it provided all the amino acid requirements of actively growing parasites and thus eliminated the need for the addition of exogenous free amino acids to the culture medium.

The composition of this medium was based upon observations suggesting an inability of $T$. cruzi to undertake de novo synthesis of purines (Fernández \& Castellani, 1958; Gutteridge \& Gaborak, 1979; Ceron et al., 1979) and various vitamins (Citri, 1954; Boné \& Parent, 1963). Using defined culture medium, however, it was demonstrated (Avila \& Avila, 1981) that T. cruzi epimastigotes do not require exogenous nucleotides for continuous cultivation, suggesting that this parasite has the capacity for de novo purine and pyrimidine synthesis. Also, only choline and folic acid, among the eight vitamins provided in the original culture medium, were necessary for discrete multiplication of $T$. cruzi.

The present paper describes an extension of this approach to the study of the continuous cultivation of seven different strains of infective epimastigotes of $T$. cruzi in a minimal medium composed only of BLC, choline, folic acid, glucose and inorganic salts. A number of features (multiplication rate, electron microscopic morphology, infectivity for vertebrate and inverte-

Abbreviations: BLC, bovine liver catalase; EPPS, 4-(2-hydroxyethyl)-1-piperazine propanesulphonic acid; LIT, liver infusion tryptose; MCRM, micromolecular component rich medium; TMM, trypanosomal minimal medium. 
brate hosts, glucose utilization and protein biosynthesis) were examined to test the nutritional quality of this new medium for supporting $T$. cruzi growth.

\section{METHODS}

Parasites. Studies were performed with strains Y, Ma, Fl, Bertoldo, A-35, Ya and EP of T. cruzi, as previously described (Avila et al., 1979a, 1981 a,b). The cultures were maintained serially in BLC-containing medium, and were used after the nineteenth passage in these experiments.

Culture media. MCRM was prepared as previously described (Avila et al., 1979a) and compared with the simplified medium for T. cruzi, TMM, the composition of which is described in Table 1. In some experiments, choline was excluded from TMM. The initial pH of both media was 6.4 and each culture flask (Falcon no. 3012, Fisher Scientific Co., Pittsburgh, Pa., U.S.A.), containing a total volume of $10 \mathrm{ml}$, received $0.3-0.8 \mathrm{ml}$ inoculum containing $5 \times 10^{6}$ parasites from the preceding subculture.

Parasite counts. At $2 \mathrm{~d}$ intervals $0.5 \mathrm{ml}$ volumes were aseptically withdrawn from the cultures at each subpassage and the parasites counted in a haemocytometer at $\times 400$ magnification.

Electron microscopy. This was carried out as previously described (Bretaña \& O'Daly, 1976).

Infectivity studies. Two different approaches were used to determine the infectivity of cultured $T$. cruzi: (1) infectivity towards vertebrate hosts using susceptible NMRI mice as previously described (Avila et al., 1979b); (2) infectivity for invertebrate hosts, using nymphs of Rhodnius prolixus. The insects were provided by the PAHO/WHO Research and Reference Centre of Vector Biology and Control, Maracay, Venezuela. They were allowed to feed for $60 \mathrm{~min}$ on each of the cultured $T$. cruzi strains contained in individual bovine intestinal membrane topped pots containing five nymphs. The insects were then maintained in a humid atmosphere at $25^{\circ} \mathrm{C}$ for 15-21 d post-feeding. The nymphs were examined individually for infection with $T$. cruzi $7-21 \mathrm{~d}$ after feeding; each nymph was wetted and killed in normal saline and the rectum and posterior intestine dissected out, separated from the rest of the gut and macerated in a small drop of saline on a microscope slide. Each preparation was thoroughly mixed and covered with a $13 \mathrm{~mm}$ diameter coverslip. The entire area under the coverslip was examined for trypanosomes using phase-contrast illumination $(\times 10$ objective, final magnification $\times 400)$.

Glucose utilization and protein biosynthesis. On withdrawal of samples for parasite counting, the $\mathrm{pH}$ was measured at room temperature $\left(20^{\circ} \mathrm{C}\right)$ before centrifugation in the cold $\left(2400 \mathrm{~g}\right.$ for $20 \mathrm{~min}$ at $\left.4{ }^{\circ} \mathrm{C}\right)$; the glucose concentration was determined in the supernatant using the anthrone method of Jermyn (1975). It has previously been demonstrated, by TLC on kieselguhr G using ethyl acetate/isopropanol/ $\mathrm{H}_{2} \mathrm{O}(65: 23: 12$, by vol.) as eluant, that glucose represents about $91-98 \%$ of the sugars present in the culture medium (unpublished observations). Protein biosynthesis was determined after $10 \mathrm{~d}$ cultivation by measuring the incorporation of $\mathrm{L}-\left[4,5-{ }^{3} \mathrm{H}\right] \mathrm{leucine}$ into soluble proteins using the method of Mancilla et al. (1967). Epimastigotes were incubated for a total of $3 \mathrm{~h}$ in the presence of $\left.\mathrm{L}-{ }^{3} \mathrm{H}\right] \mathrm{leucine}[50 \mu \mathrm{Ci}(185 \mathrm{kBq})$ per flask].

Biochemicals. BLC (C-40, lots 60F-7155 and 70F-7050, and twice crystallized C-100), glucose, streptomycin, penicillin, HEPES, EPPS and phenol red were obtained from Sigma. Folic acid, choline, the minimal essential

\section{Table 1. Composition of TMM for culture of T. cruzi}

The medium was made up to 1 litre with deionized water and the $\mathrm{pH}$ adjusted to 6.4 with $1 \mathrm{M}-\mathrm{HCl}$.

$$
\text { Component }
$$

$\mathrm{NaCl}$

$\mathrm{KCl}$

$\mathrm{MgSO}_{4} .7 \mathrm{H}_{2} \mathrm{O}$

$\mathrm{NaH}_{2} \mathrm{PO}_{4} \cdot \mathrm{H}_{2} \mathrm{O}$

$\mathrm{CaCl}_{2}$

Choline chloride

Folic acid

Glucose

$\mathrm{NaHCO}_{3}$

HEPES

EPPS

Phenol red

Streptomycin

Penicillin

Bovine liver catalase

$\begin{gathered}\text { Concentration } \\ \left(\mathrm{g} \mathrm{l}^{-1}\right)\end{gathered}$
$6 \cdot 8$
$0 \cdot 4$
$0 \cdot 2$
$0 \cdot 14$
$0 \cdot 2$
$0 \cdot 002^{*}$
$0 \cdot 002^{*}$
$1 \cdot 0$
$2 \cdot 2$
$2 \cdot 26$
$2 \cdot 34$
$0 \cdot 01$
$0 \cdot 1$
$100000 \dagger$
$3 \cdot 0$

* From a 100 -fold concentrated frozen stock solution.

$\dagger$ Concentration expressed in international units. 
medium and the vitamin mixture (100-fold concentrate) were from Grand Island Biological Co. (New York, N.Y., U.S.A.). L- $\left[{ }^{3} \mathrm{H}\right]$ Leucine $\left(1.25 \mathrm{Ci} \mathrm{mol}^{-1} ; 46.25 \mathrm{GBq} \mathrm{mol}^{-1}\right.$ ) was purchased from Schwartz-Mann (Orangeburg, N.Y., U.S.A.). Inorganic salts were obtained from Merck-Darmstadt.

\section{RESULTS}

In MCRM supplemented as described previously (Avila et al., 1979a) the seven $T$. cruzi strains studied reached an average maximum concentration of about $15 \times 10^{6}$ organisms $\mathrm{ml}^{-1}$ after $10 \mathrm{~d}$ cultivation. Strains A-35 and Ya attained the highest $\left(19 \times 10^{6}\right.$ organisms $\left.\mathrm{ml}^{-1}\right)$ and lowest $\left(7 \times 10^{6}\right.$ organisms ml-1) levels of growth in this medium respectively. These levels of growth amount to $34 \%$ of the average maximum growth obtained with a richer, undefined but conventional LIT medium (Pérez-Kepp et al., 1981). The mean growth level reached in MCRM represented a 30 -fold multiplication over the initial concentration of cells in the inoculum.

The results in Table 2 show that all seven $T$. cruzi strains grew in TMM in a continuous manner (although at different rates) for at least 13 successive passages. The Bertoldo strain gave the highest levels of growth and Ma strain gave lowest levels by comparison with the extent of growth in the control medium (MCRM). The highest average $T$. cruzi concentrations in TMM during 13 consecutive passages for all seven strains was about $9 \times 10^{6}$ organisms $\mathrm{ml}^{-1}$, which represents an 18-fold multiplication over the inoculum. The growth curves obtained in MCRM and TMM (average for seven strains) are shown in Fig. 1. Maximum growth in TMM was equivalent to $63 \%$ of that in MCRM and $20 \%$ of that in LIT medium (Pérez-Kepp et al., 1981).

No difference in $T$. cruzi growth-promoting activity was found between BLC added as purified powder (Sigma; C-40) or as twice crystallized enzyme (Sigma; C-100). However, for practical reasons, most of the experiments reported in this paper were carried out with C-40 preparations.

After several consecutive passages, $T$. cruzi strains obtained directly from MCRM were cultured in TMM under three different experimental conditions: (1) in the presence of the complete vitamin mixture used in MCRM, (2) in the presence of choline and folic acid plus only one of the other vitamins present in MCRM, (3) in the presence of folic acid alone. It was found that the individual addition of pantothenate, inositol, nicotinamide, pyridoxal, riboflavin and thiamin at the concentrations used in MCRM had no effect on parasite yield in TMM, although the addition of the total vitamin mixture allowed a marked increase in multiplication, growth being comparable to that obtained for $T$. cruzi strains routinely maintained in MCRM. Omission of choline lowered the parasite yield by about $50 \%$. These results confirmed the excellent viability of $T$. cruzi epimastigotes grown in TMM, and the importance of choline for maintaining high rates of cellular multiplication. After demonstrating the continuous multiplication of $T$. cruzi epimastigotes in TMM, we then studied the infectivity for vertebrate and invertebrate hosts of these culture forms. All ninth passage $T$. cruzi strains obtained from TMM were infective for NMRI mice and nymphs of $R$. prolixus.

\section{Table 2. Continuous growth of T. cruzi in TMM}

The composition of TMM is shown in Table 1 . Control values refer to growth obtained in MCRM, after $10 \mathrm{~d}$ cultivation at $28^{\circ} \mathrm{C}$. Values in parentheses are maximal parasite concentrations $\left(10^{6} \mathrm{cells}^{-1}\right.$ obtained in each passage in TMM).

Growth as percentage of control at passage:

$\begin{array}{lcccccrr}\text { Strain } & & 1 & 2 & 3 & 6 & 8 & 13 \\ \text { Ma } & 41(9 \cdot 1) & 52(14 \cdot 2) & 65(10 \cdot 9) & 52(11 \cdot 3) & 46(10 \cdot 2) & 58(10 \cdot 0) \\ \text { EP } & 50(9 \cdot 9) & 78(14 \cdot 5) & 85(7 \cdot 0) & 82(14 \cdot 1) & 100(11 \cdot 0) & 102(15 \cdot 0) \\ \text { FI } & 45(8 \cdot 3) & 51(12 \cdot 5) & 100(13 \cdot 9) & 56(11 \cdot 2) & 70(13 \cdot 9) & 41(8 \cdot 8) \\ \text { Y } & 24(6 \cdot 4) & 53(12 \cdot 2) & 78(11 \cdot 2) & 71(13 \cdot 8) & 55(12 \cdot 2) & 62(12 \cdot 0) \\ \text { A-35 } & 56(12 \cdot 5) & 36(9 \cdot 4) & 100(10 \cdot 4) & 36(9 \cdot 0) & 74(16 \cdot 3) & 69(14 \cdot 0) \\ \text { Ya } & 82(6 \cdot 7) & 56(5 \cdot 9) & 95(9 \cdot 9) & 59(5 \cdot 1) & 38(2 \cdot 9) & 38(2 \cdot 9) \\ \text { Bertoldo } & 100(20 \cdot 0) & 100(10 \cdot 6) & 100(14 \cdot 4) & 62(9 \cdot 4) & 55(12 \cdot 2) & 90(9 \cdot 0) \\ \quad \text { Mean } & 57 \% & 61 \% & 89 \% & 60 \% & 63 \% & 66 \%\end{array}$




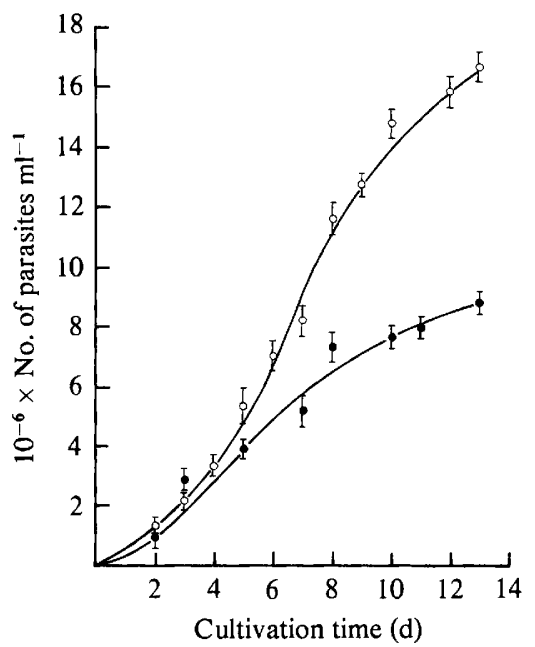

Fig. 1. Growth of T. cruzi in catalase-containing media: $\bigcirc$, MCRM;, TMM. Bovine liver catalase was at a concentration of $3 \mathrm{mg} \mathrm{ml}^{-1}$. Growth values are means from seven $T$. cruzi strains studied. The bars represent S.D.
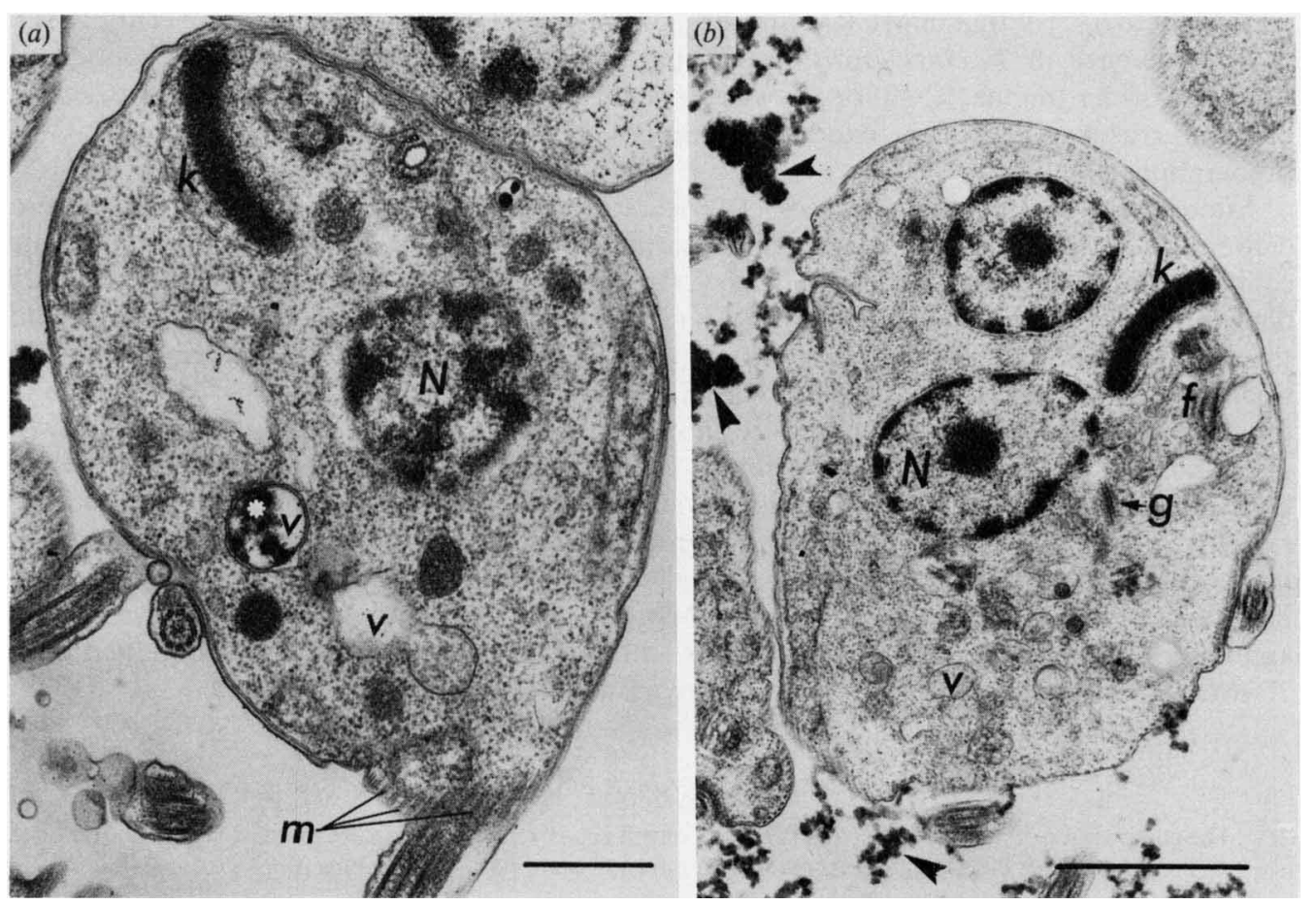

Fig. 2. Electron micrograph of longitudinal section through the kinetoplast region of $T$. cruzi, cultured in: (a) MCRM, (b) TMM. Features shown are: $\mathbf{N}$, nucleus; $f$, flagellum bases in flagellar pocket; $g$, Golgi apparatus; $m$, pellicular microtubules; $v$, inclusion vacuoles of unknown significance; and $\mathbf{k}$, kinetoplast. Bovine liver catalase aggregates can be seen outside the parasite (arrows), but also in pinocytotic vacuoles (white asterisk). The bar markers represent $1 \mu \mathrm{m}$.

The electron micrographs in Fig. 2 show some ultrastructural features of $T$. cruzi epimastigotes cultured in TMM and, for comparative purposes, in MCRM. No significant differences were observed in the ultrastructural morphology between epimastigotes cultured in either medium as compared with those grown in LIT medium (deSouza et al., 1978). 


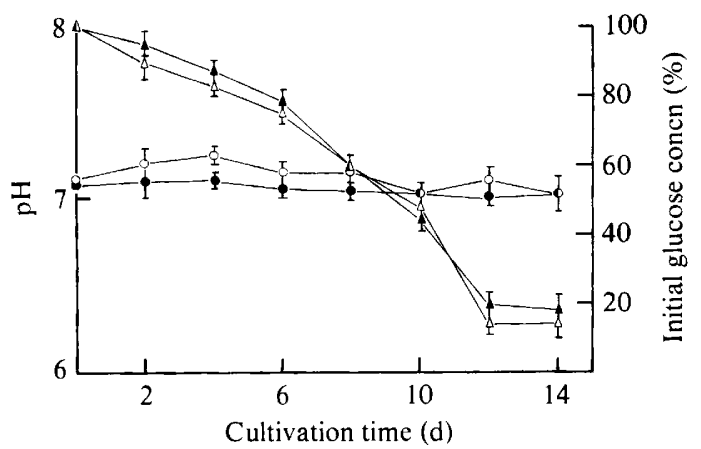

Fig. 3. pH changes $(O, \bigcirc)$ and glucose utilization $(\triangle, \Delta)$ in $T$. cruzi epimastigotes cultured in MCRM (open symbols) and TMM (filled symbols) as a function of the time of cultivation at $28^{\circ} \mathrm{C}$. Values are means from seven $T$. cruzi strains studied. The bars represent S.D.

Table 3. $\mathrm{L}-\left[{ }^{3} \mathrm{H}\right]$ Leucine incorporation into soluble proteins of $T$. cruzi

$\left[{ }^{3} \mathrm{H}\right]$ Leucine incorporation is expressed as d.p.m. (mg protein $)^{-1} \mathrm{~h}^{-1}$. Each result is the mean of 10 determinations \pm S.E. The numbers in parentheses represent the total leucine incorporation [ $\mathrm{nmol}(\mathrm{mg}$ protein $)^{-1} h^{-1}$ ] based on the specific radioactivity of the leucine.

\begin{tabular}{|c|c|c|c|}
\hline \multirow{2}{*}{$\begin{array}{l}\text { Leucine } \\
\text { concn } \\
(\mathrm{mM})\end{array}$} & \multicolumn{2}{|c|}{$\left[{ }^{3} \mathrm{H}\right]$ Leucine incorporated } & \multirow{2}{*}{$\begin{array}{c}\text { Ratio of } \\
\text { incorporation } \\
\text { TMM/MCRM }\end{array}$} \\
\hline & MCRM & TMM & \\
\hline 5 & $\underset{(5 \cdot 39)}{10500 \pm 790^{*}}$ & $\underset{(9 \cdot 45)}{22900 \pm 800^{*}}$ & $2 \cdot 18$ \\
\hline 50 & $\underset{(7 \cdot 56)}{17400 \pm} 680$ & $\begin{array}{c}26500 \pm 700 \\
(12 \cdot 01)\end{array}$ & 1.52 \\
\hline
\end{tabular}

Despite the lack of exogenous free amino acids in TMM, the glucose consumption and $\mathrm{pH}$ changes were similar to those in MCRM (Fig. 3); maximal glucose consumption was reached after $10 \mathrm{~d}$ of culture, $80 \%$ of total glucose present at the time of inoculation being consumed. This indirectly suggests that glucose utilization by the parasite is similar in both media, and that BLC, after intracellular hydrolysis, provides enough free amino acids to fulfil the nutritional requirements of actively growing epimastigotes.

The $\left[{ }^{3} \mathrm{H}\right]$ leucine incorporation into soluble proteins was significantly higher in TMM compared with MCRM (Table 3), possibly as a result of competition from other free amino acids present in the MCRM. However, in order to eliminate this possibility, $\left[{ }^{3} \mathrm{H}\right]$ leucine was also added at $10 \times$ the initial concentration. Incorporation was still found to be significantly higher in TMM, suggesting the possible existence of metabolic differences between TMM- and MCRM-cultured epimastigotes.

Finally, light microscopic analysis of the different cellular forms present in TMM revealed $96 \%$ epimastigotes and $4 \%$ trypomastigotes in the stationary phase of the culture, which compares closely to the distribution in MCRM.

\section{DISCUSSION}

Our main concern in developing a $T$. cruzi minimal medium was to find combinations of nutrient supplements to BLC which will support the continuous growth of infective parasites at a reasonably high rate. The evidence indicates that a medium composed of BLC, choline, folic acid, glucose and inorganic salts is able to support the growth of $T$. cruzi epimastigotes to an average level of about $9 \times 10^{6}$ cells $\mathrm{ml}^{-1}$ for all seven $T$. cruzi strains tested over 13 consecutive passages.

Multiplication was not further improved by addition of other vitamins ( $p$-aminobenzoic acid, biotin, pyridoxine, pyridoxamine, ascorbic acid, vitamin $B_{12}$ ) or carbohydrates (ribose, xylose, 
trehalose) (unpublished results). Also, increased concentrations of the co-factors in TMM did not give increased growth (Avila \& Avila, 1981).

Evidence was obtained of the key role of choline in supporting $T$. cruzi multiplication. Our results differed from those obtained with Crithidia fasciculata (Palmer, 1974), for which the availability of exogenous choline does not influence the rate of cellular growth. Regarding the possible role of choline in $T$. cruzi multiplication, it is known that this co-factor can serve as a precursor for phospholipid biosynthesis, and is also a labile methyl group donor.

The average maximal growth in TMM is about $20 \%$ of that attained in a conventional medium such as LIT, but this was to be expected from a defined and nutritionally poorer medium. On the other hand, the usefulness of TMM would be severely limited if the properties of the parasites, such as infectivity, morphology or metabolism, were radically changed when grown in this minimal medium. However, such problems have not materialized in our studies. Thus, ultrastructural morphology is similar to that previously reported for $T$. cruzi epimastigotes (Bretaña \& O'Daly, 1976; deSouza et al., 1978). Also, infectivity is similar to that obtained previously with cultured $T$. cruzi when tested in NMRI mice (Avila et al., 1979a), or when $R$. prolixus were fed with MCRM-derived $T$. cruzi forms. Glucose consumption was the same in both MCRM- and TMM-cultured epimastigotes, although $\left[{ }^{3} \mathrm{H}\right]$ leucine incorporation differed.

It must be stressed that neither TMM nor MCRM is able to support Trypanosoma rangeli multiplication (Avila et al., 1981b) or Leishmania brasiliensis and Leishmania mexicana proliferation (Avila et al., 1979a), suggesting that these Trypanosomatidae have different nutritional requirements. TMM, therefore, represents the first defined minimal trypanosomal medium that is useful for the specific cultivation of infective $T$. cruzi. As previously suggested (Avila et al., 1979a), BLC acted in TMM as an adequate nutritional factor, fulfilling all the amino acid requirements of actively growing parasites. It should be noted that no difference in $T$. cruzi growth-promoting activity was found between BLC used as powder (Sigma; C-40) or twice crystallized enzyme (Sigma; C-100). Furthermore, as previously reported (Avila et al., $1979 a$ ), PAGE of both C-40 and C-100 preparations of BLC revealed a major band corresponding to about $90 \%$ of the protein added to the gel and a second minor band probably corresponding to denatured protein (Sumner \& Gralén, 1938).

The fact that seven different $T$. cruzi strains (six from Venezuela and another from Brazil) grew quite well in TMM also demonstrates that we are not dealing with the cultivation of an unrepresentative 'mutant' strain.

The presence of endosymbionts has been reported in Crithidia oncopelti, Crithidia deanei and Blastocrithidia culicis (Chang, 1974; Mundim et al., 1974). In order to eliminate any possibility of contamination of our $T$. cruzi strains, serial electron microscopic and microbiological studies were undertaken, and no symbionts were found.

The authors wish to thank Dr J. Velázquez for providing $R$. prolixus nymphs, Dr Neil Lynch for comments on this manuscript and Mrs Candelaria de Aranguren for excellent secretarial services. This work was supported by CONICIT project SAL-1075.

\section{REFERENCES}

Avila, J. L. \& Avila, A. (1981). Trypanosoma cruzi: nucleotide and vitamin requirements of growing epimastigotes. Experimental Parasitology 51, 318324.

Avila, J. L., Bretaña, A., Casanova, M. A., Avila, A. \& Rodríguez, F. (1979a). Trypanosoma cruzi: defined medium for continuous cultivation of virulent parasites. Experimental Parasitology 48, 27-35.

Avila, J. L., Bretaña, A. \& Avila, A. (1979b). Chemotherapy with ethidium bromide-DNA complex in established Chagas' disease. American Journal of Tropical Medicine and Hygiene 28, 456460.

Avila, J. L., Avila, A., \& MuÑoz, E. (1981a). Effect of allopurinol on different strains of Trypanosoma cruzi. American Journal of Tropical Medicine and Hygiene 30, 795-800.

Avila, J. L., Avila, A. \& Casanova, M. A. (1981 $b$ ). Differential metabolism of allopurinol and derivatives in Trypanosoma rangeli and $T$. cruzi culture forms. Molecular and Biochemical Parasitology 4, 265-272.

Boné, G. J. \& Parent, G. (1963). Stearic acid, an essential growth factor for Trypanosoma cruzi. Journal of General Microbiology 31, 261-266.

BretaÑa, A. \& O'Daly, J. A. (1976). Uptake of fetal proteins by Trypanosoma cruzi. Immunofluorescence and ultrastructural studies. International Journal of Parasitology 6, 379-386.

Ceron, C. R., Caldas, R. A., Félix, C. R., Mundim, 
M. H. \& Roitman, I. (1979). Purine metabolism in trypanosomatids. Journal of Protozoology 26, 479 483.

Chang, K. P. (1974). Ultrastructure of symbiotic bacteria in normal and antibiotic treated Blastocrithidia culicis and Crithidia oncopelti. Journal of Protozoology 21, 699-707.

CITRI, M. (1954). Cultivation of haemoflagellates under defined conditions and analysis of the growth requirements of Trypanosoma cruzi. Ph.D. Thesis, Hebrew University, Jerusalem, Israel.

DeSouza, W., Carvalho, T. V. \& Benchimol, M. (1978). Trypanosoma cruzi: ultrastructural, cytochemical and freeze-fracture studies of protein uptake. Experimental Parasitology 45, 104-115.

Fernández, J. F. \& Castellani, O. (1958). Nucleotide and polynucleotide synthesis in Trypanosoma cruzi. I. Precursors of purine compounds. Experimental Parasitology 7, 224-235.

GutTeridge, W. E. \& GaboraK, M. (1979). A reexamination of purine and pyrimidine synthesis in the three main forms of Trypanosoma cruzi. International Journal of Biochemistry 10, 415-422.
JERMYN, M. A. (1975). Increasing the sensitivity of the anthrone method for carbohydrate. Analytical Biochemistry 68, 332-335.

Mancilla, R., Náquira, C. \& Lanas, C. (1967). Protein biosynthesis in Trypanosomidae. II. The metabolic fate of DL-leucine-1-C 14 in Trypanosoma cruzi. Experimental Parasitology 21, 154-159.

Mundim, M. H., RoItman, I., Hermans, M. A. \& KitAJIMA, E. W. (1974). Simple nutrition of Crithidia deanei, a reduviid trypanosomatid with an endosymbiont. Journal of Protozoology 21, 518-521.

Palmer, F. B. S. C. (1974). Biosynthesis of choline and ethanolamine phospholipids in Crithidia fasciculata. Journal of Protozoology 21, 160-163.

Pérez-KePp, R., Avila, J. L. \& Bretaña, A. (1981). Comparative study of different Trypanosoma cruzi strains cultured in three different media. Acta cientifica venezolana 32 (suppl.), 144.

Sumner, J. B. \& Gralén, N. (1938). The molecular weight of crystalline catalase. Journal of Biological Chemistry 125, 33-36. 